\title{
Anisakiasis gastro-alérgica, primera descripción de un caso en Colombia y revisión bibliográfica
}

\author{
John A. Patiño', Mario J. Olivera² \\ ${ }^{1}$ Facultad de Medicina, Universidad Nacional de Colombia, Bogotá, D.C., Colombia \\ ${ }^{2}$ Grupo de Parasitología, Instituto Nacional de Salud, Bogotá, D.C., Colombia
}

La anisakiasis es una enfermedad parasitaria zoonótica causada por el consumo de pescados o mariscos crudos o poco cocidos infectados con nematodos de los géneros Anisakis, Pseudoterranova y Contracaecum.

Se describe el primer caso de anisakiasis en Colombia y se resume la literatura médica disponible. Una mujer de 52 años de edad consultó por dolor epigástrico agudo de inicio abrupto, náuseas, vómitos, diarrea y urticaria después de consumir pescado.

El examen físico reveló sensibilidad moderada en el epigastrio. El examen de laboratorio evidenció leucocitosis, en tanto que la radiografía simple y el electrocardiograma no reflejaron ninguna anormalidad. El diagnóstico se hizo mediante una endoscopia de vías digestivas altas, la cual reveló engrosamiento de la pared gástrica y un parásito en movimiento. Se encontró una larva de Anisakis y se la extrajo por endoscopia, lo que alivió el dolor de la paciente.

Clínicamente, la anisakiasis puede presentarse como una enfermedad gástrica, intestinal, en otros sistemas o alérgica. El diagnóstico se hace con base en la elaboración del historial alimentario del paciente y la visualización directa de las larvas; el único tratamiento efectivo consiste en su extracción endoscópica.

Palabras clave: Anisakis; nematodos; enfermedades transmitidas por alimentos; hipersensibilidad; enfermedades gastrointestinales; Colombia.

\section{Gastro-allergic anisakiasis: The first case reported in Colombia and a literature review}

Anisakiasis is a zoonotic parasitic disease caused by consumption of raw or undercooked fish or seafood infected with nematodes of the Anisakis, Pseudoterranova or Contracaecum genera.

Here, we describe the first case of anisakiasis in Colombia and summarize the available

Recibido: 01/08/17

Aceptado: 03/09/18

Publicado: 04/09/18

Citación:

Patiño JA, Olivera MJ. Anisakiasis gastro-alérgica, primera descripción de un caso en Colombia y revisión bibliográfica. Biomédica. 2019;39:241-6. https://doi.org/10.7705/biomedica.v39i2.3936

\section{Correspondencia:}

Mario J. Olivera, Grupo de Parasitología, Instituto Nacional de Salud, Calle 26 № 51-20, Bogotá, D.C., Colombia

Teléfono: (51) 220 7700, extensión 1223

moliverajr@gmail.com

\section{Contribución de los autores:}

John A. Patiño: recolección de datos y discusión de los hallazgos clínicos

Mario J. Olivera: concepción y diseño de

manuscrito, y manejo clínico del paciente

Ambos autores participaron en el análisis y la interpretación de los datos, y la redacción del manuscrito.

Financiación:

Para la realización de este trabajo no se requirió financiación.

\section{Conflicto de intereses:}

Los autores declaramos que no existió ningún tipo de conflicto de intereses en la realización del presente trabajo. literature. A 52-year-old female with a history of abrupt-onset sharp epigastric pain, nausea, vomit, diarrhea, and urticaria following fish consumption consulted the health service. The physical examination revealed moderate tenderness of the epigastric region; the laboratory evaluation showed leukocytosis and a simple X-ray and ECG showed no abnormalities. The diagnosis was made by endoscopic examination, which revealed a thickened gastric wall and a moving larval worm. An Anisakis larva was found and extracted endoscopically, which relieved the pain of the patient.

Clinically, anisakiasis may present as a gastric, intestinal, extragastrointestinal or allergic disease. Diagnosis and treatment of anisakiasis are made by a dietary history, direct visualization and endoscopic extraction of possible larvae, which is the only effective therapy.

Keywords: Anisakis; nematoda; foodborne diseases; hypersensitivity; gastrointestinal diseases; Colombia.

La anisakiasis, reportada por primera vez en Holanda en 1955 por van Thiel (1), es una zoonosis accidental causada por la ingestión de pescado crudo o poco cocido infestado con larvas infectivas en estadio L3 del nematodo Anisakis simplex.

Se trata de una enfermedad cosmopolita que tiene mayor incidencia en aquellas regiones donde el pescado crudo hace parte de la gastronomía, como sucede en los países asiáticos. Sin embargo, debido a la expansión de este tipo de preparaciones, es posible encontrarla en muchos otros lugares. Además, la infestación puede aparecer por el consumo de pescados con cocción o refrigeración deficiente. Se presenta el primer reporte de este parásito en humanos en Colombia. 


\section{Presentación del caso}

Se trata de una profesora de 52 años de edad procedente de Bogotá, que presentó un cuadro clínico de dos días de evolución consistente en epigastralgia intensa, náuseas, vómitos y diarrea, así como inapetencia y urticaria. No presentó fiebre.

La paciente refirió que los síntomas se habían iniciado después de ingerir alimentos a base de pescado crudo en una zona costera del departamento de Córdoba, Colombia. En su caso, el consumo de pescado crudo era tradición familiar.

En el examen físico se evidenció palidez mucocutánea generalizada y dolor en el mesogastrio con la palpación. Se le hizo un hemograma en el que se evidenció leucocitosis con neutrofilia y eosinofilia. En el electrocardiograma y la radiografía de tórax, no se observaron alteraciones. La endoscopia de vías digestivas altas permitió detectar un verme móvil, serpenteante, con un extremo aferrado a la mucosa del antro; el área alrededor estaba edematosa y eritematosa.

El verme se extrajo y se fijó en glutaraldehído para luego ser enviado al Instituto Nacional de Salud, en donde se hizo el análisis parasitológico. En este se describió una larva de $A$. simplex (s.l.) de $35 \mathrm{~mm}$ de longitud y $0,6 \mathrm{~mm}$ de ancho, de color blanco, con tres labios bilobulados en la región cefálica, un diente perforador ventral, glándulas excretoras anteriores, estómago largo, sin ciego, y cola terminada en punta roma. El resultado del coprocultivo ordenado fue normal.

La paciente refirió mejoría de la sintomatología gastrointestinal y alérgica después de la extracción del parásito. A pesar de que el resultado del coprocultivo fue negativo para parasitosis, se consideró necesario administrar una dosis única de $400 \mathrm{mg}$ de albendazol.

\section{Discusión}

El término 'anisakiasis' se refiere a la infestación por $A$. simplex (s.l.), aunque también se utiliza el término "anisakidosis" para referirse a la producida por diferentes especies de la familia Anisakidae, como Pseudoterranova decipiens (s.l.), Contracaecum osculatum (s.l.) y A. simplex (s.l.); estos dos términos conviven en la literatura especializada de forma indistinta (2).

Los ejemplares adultos de $A$. simplex (s.I.) miden de 2 a $3 \mathrm{~cm}$ de largo por $1 \mathrm{~cm}$ de diámetro, y se encuentran normalmente en cetáceos (ballenas y delfines), en cuyo interior alcanzan la madurez sexual adheridos a la mucosa gastrointestinal y luego expulsan los huevos sin embrión, los cuales alcanzan el agua junto con las heces de sus huéspedes.

Una vez en el agua, se forman los embriones y las larvas evolucionan hasta el estadio L2, caracterizado por ser de vida libre. Posteriormente, las larvas son ingeridas por crustáceos (principalmente copépodos y eufausiáceos), en los cuales pasan al estadio L3 y adoptan la forma infectiva para peces y calamares.

En estos, que actúan como huéspedes paraténicos o de transporte, las larvas L3 migran del intestino hacia los tejidos de la cavidad peritoneal y crecen hasta $3 \mathrm{~cm}$ de largo y son infectivas. También, pueden migrar al tejido muscular, enquistarse, permanecer en estado latente y pasar de pez en pez 
mediante la depredación. Cuando los mamíferos marinos ingieren estos peces o calamares infestados con larvas L3 estas mudan dos veces, se convierten en parásitos adultos y producen huevos que se esparcen en el agua $(3,4)$.

El ser humano se infesta cuando consume peces con larvas $L 3$, sin la debida congelación o cocción (figura 1). El equivalente de la anisakiasis en peces es la ascariasis o ascaridiasis (5).

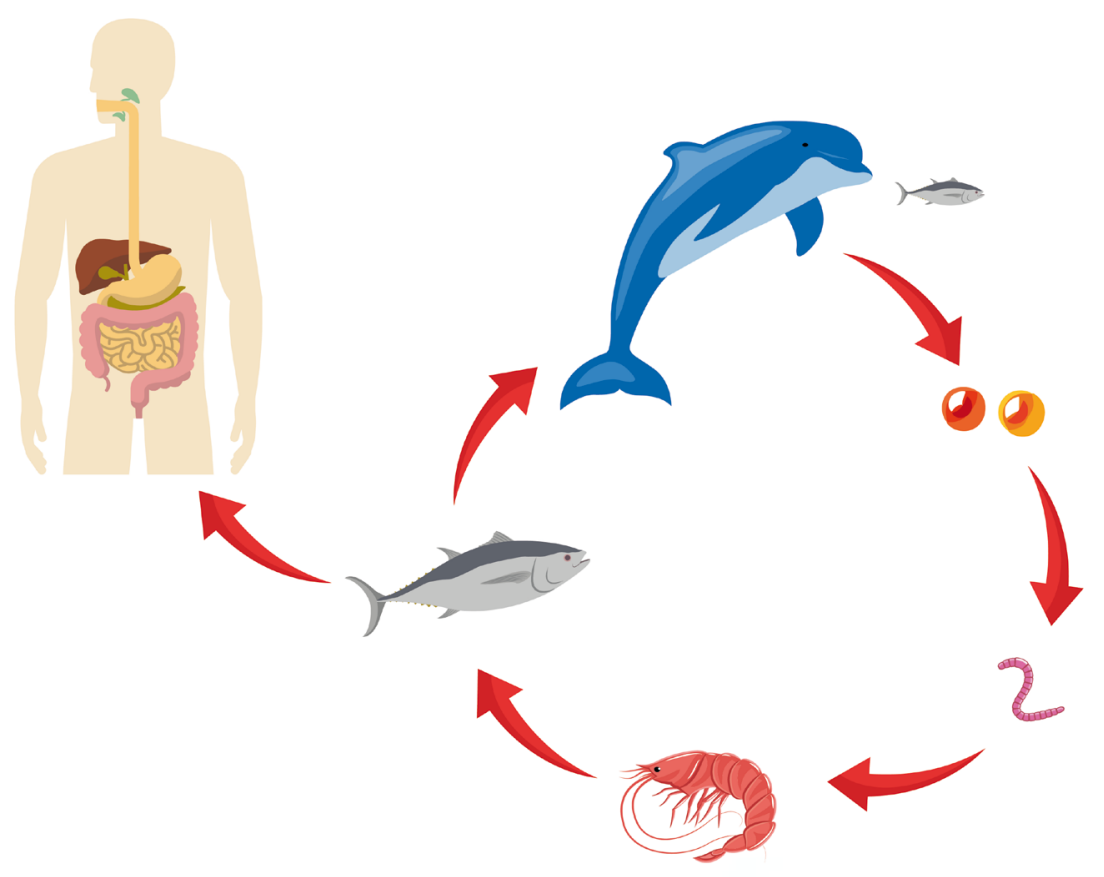

Figura 1. Esquema del ciclo biológico de Anisakis simplex

En su etapa de adulto, Anisakis simplex parasita una gran variedad de mamíferos marinos, incrustándose en la mucosa del estómago. Los huevos sin embrión son expulsados por las hembras adultas y llegan al medio marino con las heces de los huéspedes definitivos.

Dentro del huevo se desarrollan la primera (L1) y la segunda etapas larvarias (L2), luego eclosionan y producen larvas de vida libre que son ingeridas por los crustáceos (huésped intermediario). Las larvas ingeridas se convierten en larvas L3, las cuales son infectivas para peces y calamares (huéspedes paraténicos). Las larvas migran del intestino a los tejidos de la cavidad peritoneal y crecen hasta los $3 \mathrm{~cm}$ de longitud. Tras la muerte del huésped, las larvas migran a sus tejidos musculares y se transfieren de un pez a otro. El ciclo vital se completa cuando un mamífero marino (cetáceo) se alimenta de un animal infestado. Los seres humanos se infestan accidentalmente cuando ingieren pescado crudo o poco cocinado. Después de la ingestión, las larvas de Anisakis penetran la mucosa gástrica e intestinal y causan los síntomas de la anisakiasis.

Una vez ingeridas las larvas $L 3$, se producen las manifestaciones clínicas mediante dos mecanismos: el primero es la hipersensibilidad inmediata mediada por lgE frente a los antígenos de $A$. simplex, entre ellos los somáticos, los de excreción y los de secreción, los cuticulares y los del fluido seudocelómico. El segundo mecanismo es el daño directo causado por el nematodo en el segmento del tubo digestivo donde se asienta (6).

Las reacciones alérgicas pueden ir desde urticaria, angioedema 0 broncoespasmo, hasta la anafilaxia. El efecto local se presenta como dolor abdominal leve a intenso, náuseas, vómitos o diarrea que, en algunos casos, conducen a la expulsión natural del parásito $(7,8)$. Además, se ha estimado que, aproximadamente, un tercio de los nematodos ingeridos puede afectar sistemas distintos al gastrointestinal al perforar la pared del tubo digestivo y migrar a otras localizaciones, como la mucosa esofágica y la orofaríngea, la cavidad abdominal, el epiplón mayor y el mesenterio (4). 
Asimismo, se ha señalado que esta parasitosis es un factor de riesgo para el desarrollo de cáncer gastrointestinal debido a la inflamación crónica y al posible efecto cancerígeno de ciertas secreciones parasitarias; además, se han reportado hallazgos incidentales de la larva en tumores gastrointestinales $(9,10)$.

Los síntomas de la anisakiasis son variados e inespecíficos, por lo cual en muchos países no se diagnostica oportunamente. En nuestro medio, esta parasitosis no suele reconocerse, lo que puede aumentar el subdiagnóstico. En 1967, en un estudio clínico-patológico de 92 casos de anisakiasis en Japón, se reportó que más del $60 \%$ de ellos se diagnosticaron como hallazgo quirúrgico durante intervenciones por apendicitis, abdomen agudo, tumor gástrico o cáncer, ileítis, colecistitis o diverticulitis, entre otras (11). Aún hoy, la anisakiasis sigue siendo comúnmente diagnosticada como apendicitis o enfermedad de Crohn (12).

El diagnóstico se presume a partir de la sintomatología y el antecedente de haber consumido pescado crudo o poco cocido, y se confirma mediante la endoscopia digestiva, que es la herramienta más útil, ya que permite la visualización directa de las larvas en el tubo digestivo y, además, facilita su cuantificación para extraerlas todas.

Existen otros medios diagnósticos, como la medición de los niveles de IgE total, los cuales aumentan debido a los mecanismos de hipersensibilidad dependientes de IgE que se ponen en marcha con la infestación. No obstante, esta prueba no es específica, ya que son varias las causas que pueden desencadenar los síntomas y elevar la concentración de lgE total. También, existe la posibilidad de medir los niveles específicos de lgE contra antígenos propios de los parásitos mediante ELISA, pero este método no está disponible comercialmente en Colombia. Es frecuente encontrar en el hemograma leucocitosis con neutrofilia y eosinofilia (13).

El tratamiento consiste en remover las larvas durante la endoscopia gastrointestinal, lo cual alivia los síntomas en pocos días (13-15). En algunos casos, cuando los vermes se localizan por fuera del tubo digestivo, se puede requerir tratamiento quirúrgico para extraerlos. El manejo de los síntomas de hipersensibilidad es el mismo que el de otras alergias, es decir, con antihistamínicos o glucocorticoides.

En casi todos los mares del mundo se ha reportado la presencia de larvas de $A$. simplex (s.l.) en las piezas capturadas; la parasitación en peces afecta entre el 40 y el $80 \%$ de los especímenes, dependiendo de la especie del pez y de su procedencia (16). Pardo, et al., demostraron que la prevalencia de la parasitosis con nematodos del género Contracaecum era mayor del $95 \%$ en los peces rubios de los ríos Sinú y San Jorge en Colombia (17). También, se ha demostrado la presencia de peces parasitados por este nematodo en otros lugares del país (18-20), por lo cual la probabilidad de que la infestación humana se presente es alta.

La anisakiasis se puede prevenir asegurando la congelación a $-20^{\circ} \mathrm{C}$ de los pescados durante un periodo mínimo de 24 horas o su cocción a $60^{\circ} \mathrm{C}$ durante 10 minutos. El número de casos de infestación en los Países Bajos se redujo casi a cero con la inclusión de estas medidas en la legislación (21).

Después de una búsqueda de artículos sobre la anisakiasis humana en Colombia, se pudo determinar que este es el primer caso diagnosticado. Hay que concienciar y divulgar información sobre esta enfermedad, para que se pueda diagnosticar y tratar exitosamente. 


\section{Conclusiones}

Este es el primer reporte de anisakiasis humana en Colombia y pone de manifiesto la necesidad de ampliar el estudio de este tipo de enfermedades. La anisakiasis se presenta con diversos síntomas poco específicos, lo que dificulta una rápida impresión diagnóstica que conduzca a su detección, por lo que los hallazgos son incidentales. El antecedente de haber consumido pescado crudo o poco cocido es importante para sospecharla. La implementación de medidas para el manejo del pescado antes de su consumo puede prevenir la infestación con estos parásitos.

\section{Referencias}

1. Valls A, Pascual CY, Martín-Esteban M. Anisakis y anisakiosis. Allergol Immunopathol (Madr). 2003;31:348-55. https://doi.org/10.1016/S0301-0546(03)79211-8

2. Baird FJ, Gasser RB, Jabbar A, Lopata AL. Foodborne anisakiasis and allergy. Mol Cell Probes. 2014;28:167-74. https://doi.org/10.1016/j.mcp.2014.02.003

3. Centers for Disease Control and Prevention. Anisakiasis. Fecha de consulta: 10 de abril de 2017. Disponible en: https://www.cdc.gov/parasites/anisakiasis/biology.html

4. Mattiucci S, Cipriani P, Paoletti M, Levsen A, Nascetti G. Reviewing biodiversity and epidemiological aspects of anisakid nematodes from the North-east Atlantic Ocean. $J$ Helminthol. 2017;91:422-39. https://doi.org/10.1017/S0022149X1700027X

5. Hochberg NS, Hamer DH. Anisakidosis: Perils of the deep. Clin Infect Dis. 2010;51:806-12. https://doi.org/10.1086/656238

6. López D, Ramírez LM, del Rosal R, López F, Fernández-Crehuet R, Miño G. Anisakiasis in Spain: An increasing disease. Gastroenterol Hepatol. 2000;23:307-11.

7. Nieuwenhuizen NE, Lopata AL. Allergic reactions to Anisakis found in fish. Curr Allergy Asthma Rep. 2014;14:455. https://doi.org/10.1007/s11882-014-0455-3

8. Shimamura Y, Muwanwella N, Chandran S, Kandel G, Marcon N. Common symptoms from an uncommon infection: Gastrointestinal anisakiasis. Can J Gastroenterol Hepatol. 2016;2016:5176502. https://doi.org/10.1155/2016/5176502

9. García-Pérez JC, Rodríguez-Pérez R, Ballestero A, Zuloaga J, Fernández-Puntero B, AriasDíaz J, et al. Previous exposure to the fish parasite Anisakis as a potential risk factor for gastric or colon adenocarcinoma. Medicine. 2015;94:e1699. https://doi.org/10.1097/MD.0000000000001699

10. Sonoda H, Yamamoto K, Ozeki K, Inoye H, Toda S, Maehara Y. An Anisakis larva attached to early gastric cancer: Report of a case. Surg Today. 2015;45:1321-5. https://doi.org/10.1007/s00595-014-1012-3

11. Yokogawa M, Yoshimura H. Clinicopathologic studies on larval anisakiasis in Japan. Am J Trop Med Hyg. 1967;16:723-8. https://doi.org/10.4269/ajtmh.1967.16.723

12. Montalto M, Miele L, Marcheggiano A, Santoro L, Curigliano V, Vastola M, et al. Anisakis infestation: A case of acute abdomen mimicking Crohn's disease and eosinophilic gastroenteritis. Dig Liver Dis. 2005;37:62-4. https://doi.org/10.1016/j.dld.2004.05.014

13. Barriga J, Salazar F, Barriga E. Anisakiasis: presentación de un caso y revisión de la literatura. Rev Gastroenterol Perú. 1999;19:317-23.

14. Henríquez A, Villafruela M. Anisakis: pasado, presente y futuro. Med Clin. 2009;132:400-3. https://doi.org/10.1016/j.medcli.2008.11.022

15. Fuchizaki U, Nishikawa M. Gastric anisakiasis. N Engl J Med. 2016;375:e11. https://doi.org/10.1056/NEJMicm1509451

16. Colombo F, Cattaneo P, Castelletti M, Bernardi C. Prevalence and mean intensity of Anisakidae parasite in seafood caught in the Mediterranean sea focusing on fish species at risk of being raw-consumed. A meta-analysis and systematic review. Crit Rev Food Sci Nutr. 2016;56:1405-16. https://doi.org/10.1080/10408398.2012.755947

17. Pardo CS, Mejía PK, Navarro VY, Atencio GV. Prevalencia y abundancia de Contracaecum sp. en rubio Salminus affinis en el río Sinú y San Jorge: descripción morfológica. Rev MVZ Córdoba. 2007;12:887-96. https://doi.org/10.21897/rmvz.433 
18. Olivero-Verbel J, Baldiris-Avila R, Arroyo-Salgado B. Nematode infection in Mugil incilis (Lisa) from Cartagena Bay and Totumo Marsh, north of Colombia. J Parasitol. 2005;91:110912. https://doi.org/10.1645/GE-392R1.1

19. Castellanos JA, Tangua AR, Salazar L. Anisakidae nematodes isolated from the flathead grey mullet fish (Mugil cephalus) of Buenaventura, Colombia. Int J Parasitol Parasites Wildl. 2017;6:265-70. https://doi.org/10.1016/j.ijppaw.2017.08.001

20. Olivero-Verbel J, Baldiris-Ávila R, Güette-Fernández J, Benavides-Álvarez A, MercadoCamargo J, Arroyo-Salgado B. Contracaecum sp. infection in Hoplias malabaricus (moncholo) from rivers and marshes of Colombia. Vet Parasitol. 2006;140:90-7. https://doi.org/10.1016/j.vetpar.2006.03.014

21. Nieuwenhuizen NE, Lopata AL. Allergic reactions to Anisakis found in fish. Curr Allergy Asthma Rep. 2014;14:455. https://doi.org/10.1007/s11882-014-0455-3. 